patients with AISC had UC and 2 IBDU. 40\% of patients had simultaneous diagnosis of AILD and IBD, all presented with symptoms of bowel disease and abnormal GGT and/or aminotransferase activity (6/10 had PR bleeding). 15 (60\%) patients were diagnosed with AILD and concomitant IBD after 19 months (mean time). In 7/15 gut symptoms improved since immunomodulators started but FC was raised, $3 / 15$ had no gut symptoms but raised FC on screening and $5 / 15$ developed bowel symptoms after liver diagnosis, in 3 of them FC was raised since liver diagnosis. Endoscopic features included pancolitis in $60 \%$ and ileitis in 20\%. Histological features were more consistent with those of mild to moderate UC (78\%). All patients diagnosed with AILD were started on steroids, 9/25 were already on Azathioprine before the endoscopic assessment for IBD.

Conclusions $20 \%$ of patients with primary diagnosis of AILD had IBD. 40\% had simultaneous diagnosis; all had raised FC since AILD was identified. We recommend FC routinely in children with AILD for the early diagnosis of IBD. Colonoscopy should be considered in patients with symptoms of IBD and the ones with clearly elevated FC. The timing of the assessment is of paramount as immunosuppresive treatment can mask symptoms and change the disease activity.

\section{P47 SETTING UP A REGIONAL HOME CALPROTECTIN SERVICE DURING THE COVID-19 PANDEMIC OFFERING HOSPITAL-BASED TESTING TO LOCAL AND REGIONAL PAEDIATRIC IBD PATIENTS IN WESSEX}

Claire Barnes, Chris Roberts, Jo Ward, Tracy Coelho, Akshay Batra, Robert Mark Beattie, Efrem Eren, Nadeem Afzal. University Hospital Southampton

\subsection{6/flgastro-2021-bspghan.56}

Background The first wave of the COVID-19 pandemic in the UK severely restricted our regional paediatric GI outpatient services affecting our ability to assess patients in hospital, further compounded by distance of travel of patients (An audit form 2019 showed $70 \%$ of patients endoscoped were from outside the local, rather than Southampton area). The issue was further compounded by some DGH's, who stopped offering the calprotectin test due to COVID-19 infection risk to the staff. Although home based calprotectin kits are also available, families using them have reported their use cumbersome and difficult to process tests at home. In addition, calprotectin results from other laboratories may be difficult to access. These limitations led to the development of a new regional service, in which samples taken at home are posted to the hub hospital laboratory (where the IBD clinic is based) for Calprotectin testing.

Aim To study the benefits of offering a service for posting faecal samples for calprotectin testing to a hub laboratory.

Methods Children (0-18 years) with IBD in the Wessex region, UK needing a calprotectin test were given postal faecal calprotectin packs (PFCP), either by hand in clinic or posted to their home. Each PFCP contained a labelled specimen bottle with immunology request form, bio-packaging box, sealable return bag (UN3373 compliant) with attached freepost label and instruction sheet. A Calprotectin cut off level of $<200$ was used as normal.

Results 63 patients $(M=34,54 \% \& F=29,46 \%)$ were given PFCP between 27th July \& 5th of Nov 2020 with $52.4 \%$ posted PFCP and $47.6 \%$ given PFCP by hand in the paediatric GI clinic. The patients resided at a mean distance of 41.6 miles (1 SD $=24.1$ miles) as the crow flies from the hospital. A mean of 25 days $(1 \mathrm{SD}=10$ days) were taken from posting/handing of PFCP to the lab test result being obtained.

The PFCP was returned by 50 patients (79.4\% compliance) with a diagnosis of Crohn's disease 34.9\%, UC 28.6\%, IBDU $7.9 \%$, oral ulcers $4.8 \%$ and $23.8 \%$ of patients referred for endoscopy with IBD like symptoms. $30 \%$ of the patients with IBD (15/50) posting the PFCP had an abnormal test result. This led to a change in management in $40 \%$ of the patients. In the patient group referred with suspected IBD only $1 / 15$ patients had an abnormal calprotectin test. $70 \%$ of patients with a normal test were able to be reassured without further investigation.

Conclusion This is the first reported series, offering to a large region a robust method for samples to be taken at home and posted to a central hub laboratory for calprotectin testing during the COVID-19 pandemic. Test results were readily available, being performed in the same hospital site as the IBD clinic. Compliance with the new PFCP remains high with $80 \%$ using the new PFCP service, with value in early identification of patients who may not have much in terms of symptoms and avoidance of endoscopy in others with a normal calprotectin.

\section{P48 $\quad$ SHOCK COLON}

${ }^{1}$ Milda Jancauskaite, ${ }^{2}$ Alison Campbell, ${ }^{2}$ Bruce Jaffray, ${ }^{1}$ Sean Marven. 'Department of Paediatric Surgery, Sheffield Children's Hospital; ${ }^{2}$ Department of Paediatric Surgery, Great North Children's Hospital, Newcastle

10.1136/flgastro-2021-bspghan.57

Introduction Non-occlusive mesenteric ischaemia (NOMI) is rare in children. There are individual case reports of ischaemic colitis, with various underlying causes for the acute deterioration. The likely mechanism is hypoperfusion/reperfusion injury. The outcome tends to be poor. Potentially; because the initial hit to the whole system is so significant, or possibly because of the toxicity of the colonic insult. Promptness of colonic resection does not seem to improve survival.

In our institutions we recognised a series of critically ill patients with a similar pattern of colonic injury. The patients had a comparable clinical picture and outcome. Therefore, we hypothesised; a similar underlying pathophysiology might be responsible.

By collaborating, the expectation was that we would identify key learning points.

Aim We aimed to identify patterns in presentation and correlate these with, surgical and pathology findings. By studying our cohort and reviewing the existing literature, we hoped to identify a possible means of improving survival.

Subject and Methods We reviewed clinical notes, histology, radiology and laboratory results of 4 consecutive cases of idiopathic colonic gangrene associated with acute cardiovascular collapse. Patients presented over a 2-year period to our 2 institutions (both providing tertiary paediatric surgery). We reviewed the literature on young adults and children with ischaemic colitis and non-occlusive mesenteric ischaemia. 